\title{
Les obstacles au succès de l'innovation organisationnelle et managériale dans les Petites et Moyennes Entreprises du secteur du Bâtiment et des Travaux Publics au Burkina Faso
}

\author{
SOME Lucain \\ Université Aube Nouvelle - Ouagadougou- Burkina Faso
}

\begin{abstract}
Résumé : La présente recherche est basée sur une démarche qualitative s'appuyant sur l'exploitation de de données secondaires. L'objectif de la recherche est de déterminer les facteurs susceptibles d'influencer sur le succès de l'innovation dans les Petites et Moyennes Entreprises (PME) du Bâtiment et des Travaux Publics (BTP) au Burkina Faso. Pour ce faire, nous avons émis deux propositions de recherche qui ont servi de fils conducteur. Il est ressorti que 77\% des PME ont déclaré avoir innové en 2019 (SOME, 2019). Alors que la même étude a conclu à la contre-performance de ces entreprises. D'où la nécessité de faire la distinction entre les actions d'innovation et le succès de l'innovation. Pour nous le succès de l'innovation repose sur la transparence informationnelle dans l'entreprise et la valorisation des ressources humaines. Les résultats indiquent qu'il manque une transparence informationnelle dans les entreprises du BTP. Aussi, les PME du BTP manquent du personnel compétent, qualifié et sont peu motivés. Du reste le manque de personnel compétent et motivé est confirmé par les Maîtres d'ouvrage (MO) et Maîtres d'Ouvrage Délégué (MOD). Nous deux propositions de recherche ont été validées.

Cependant, nous avons noté qu'il s'agit d'une recherche partielle des facteurs qui influencent sur le succès de l'innovation dans les PME du BTP qui pourraient être complétés par d'autres recherches.
\end{abstract}

Mots clés : PME du BTP, Innovation organisationnelle et managériale, capacité dynamique. 


\section{Introduction}

La création de la richesse en Afrique est une question d'actualité. Elle l'a d'ailleurs toujours été depuis les indépendances, notamment francophone porté jusque-là à des coûts de subventions par la métropole colonisatrice (Sylla, 2013).

Face à l'incapacité de l'Etat de pouvoir à l'emploi de tous les demandeurs, les Etats africains ont fait du secteur privé un moteur en matière de création d'emploi (SOME, 2016). Cela se traduit par la mise en place de diverses structures d'appui à la création des entreprises, cas de la création de la maison de l'entreprise en 2006 (MEB). Ainsi, les actions conjuguées de l'Etat et de ses partenaires ont permis d'accroitre substantiellement le nombre moyen annuel de création d'entreprise. Au total, ce sont 35400 entreprises ont été créées à travers la MEB entre 2006 et 2013 soit huit (8) années d'existence (MEB, 2015). Dans ce nombre, il y a une prédominance des entreprises individuelles, soit 80\% (MEB, 2015).

Cependant, leur contribution à la résorption du chômage demeure très faible, 2 entreprises sur 3 emploiyent moins de 10 personnes (INSD, RSE 2010). Cette faible contribution s'explique par le fait que les entreprises rencontrent d'énormes difficultés dès leur création. Les conclusions de l'étude réalisée en 2014 sur le profil de mortalité des entreprises présentent trois cas des entreprises créées. Primo, il y a un décalage important entre la date de création de l'entreprise et celle de démarrage réel des activités. Secondo, le taux de morbidité des entreprises est très élevés, soit $28,31 \%$ qui disparaissent avant leur $3^{\text {ème }}$ anniversaire et tertio, celles qui résistent dans le temps font preuve d'une grande stagnation : il se pose alors le problème de la performance des PME au Burkina Faso.

En effet, l'une des principales critiques généralement formulées à l'endroit des micro et petites entreprises est la faiblesse de leur développement (Sylla, 2013). Déjà en 2006, le rapport des nations unies indiquaient que seulement $7 \%$ des micro entreprises se transforment en moyenne ou grandes entreprises, la plupart des entreprises conservant leur taille. C'est au vu de cette faiblesse d'évolution des entreprises que les chercheurs se sont intéressés à leur performance. La performance, bien que polysémique, peut être considérée comme la résultante d'un compromis dynamique, évolutif et équilibré entre quatre pôles complémentaires de contraintes : les ressources économiques, les ressources humaines, les ressources techniques et organisationnelles et les ressources règlementaires (lois, règles, normes volontaires), (Bonnin et Bedr, 2001). Au regard de ces contraintes, pour résister au temps, l'entreprise doit développer des stratégies afin de transformer ces contraintes en opportunité : il s'agira donc pour l'entreprise de s'engager dans une dynamique d'innovation. 
En effet, l'innovation est un facteur déterminant de la compétitivité et de la rentabilité des entreprises ; elle est par conséquent un élément essentiel de la stratégie de l'entreprise.

$\mathrm{Au}$ regard de ces quatre facteurs, sur quoi alors l'innovation doit porter afin d'assurer la pérennité de l'entreprise. La littérature fait ressortir plusieurs dimensions de l'innovation (technologique, de produits, de processus etc.).

Dans le cadre de cette recherche, nous avons retenu l'innovation organisationnelle et managériale (IOM).

\section{Problématique}

L'innovation est un vaste domaine de recherche qui intéresse plusieurs disciplines des sciences sociales (Ayerbe-Machat, 2003). Dans un contexte concurrentiel et face aux perpétuels changements de leur environnement, les entreprises mènent une réflexion sur l'innovation qui est essentielle pour assurer leur croissance et leur développement.

Selon deux recherches effectuées en 2016 et $2019^{1}$ auprès des entreprises du BTP, il est ressorti respectivement que $36 \%$ et $77 \%$ des entreprises ont déclaré avoir innové les trois dernières années. Il apparait que les entreprises ont conscience que leur survie dépend de leur capacité d'innovation.

Cependant, malgré ce fort taux, on constate que le taux de mortalité des entreprises demeure élevé, et donc leur performance mise en cause. Il se pose alors la problématique du succès des innovations dans les entreprises du BTP.

Aussi, la recherche a-t-il montré que 33\% des chefs d'entreprises ne sont pas satisfaits de leur part de marché (SOME, 2019). Les croisements de variables effectués entre la satisfaction des parts de marché et l'innovation ont montré une forte influence réciproque des deux variables. En effet, 60,9\% des entreprises qui ont innové ces trois (3) dernières années, sont satisfaites du niveau de leur chiffre d'affaires contre $85,7 \%$ qui n'ont pas innové et qui ne sont pas satisfaites de leur chiffre d'affaires (SOME 2019).

Nous avons donc conclu qu'il y a une influence entre l'innovation et certaines variables de l'entreprise, notamment l'augmentation des parts de marchés.

Pour nous la réussite de toute entreprise passe par son organisation et son système de management, d'où le choix d'aborder l'innovation dans le sens de l'organisation et du management.

\footnotetext{
${ }^{1}$ Il s'agit d'une étude réalisée auprès de 26 PME du BTP dans le cadre d'un Master de recherche et la deuxième dans le cadre d'une thèse. Ces différents taux s'expliquent par le fait que la première étude a pris en compte les TPE et PME alors que la deuxième étude a concerné uniquement les moyennes entreprises
} 
Aussi, il nous parait nécessaire d'encadrer notre démarche à travers une question de recherche autour de laquelle nous allons mener nos investigations. Cette question est la suivante :

Quels sont les obstacles au succès de l'innovation organisationnelle et managériale dans les PME du BTP au Burkina Faso ?

Il s'agit donc pour nous à travers cette question de recherche d'identifier quelques variables susceptibles d'influencer significativement sur le succès des actions d'innovation organisationnelles et managériales dans les entreprises du BTP. Mais avant, il est indispensable de préciser en quoi ce thème présente un intérêt pour le monde de la recherche.

\section{Originalité du thème}

Toute recherche doit apporter une contribution au progrès de la science, de ce fait doit présenter non seulement une certaine originalité qui permet d'aboutir à des résultats pertinents. Ainsi, les PME représentent un maillon important pour l'économie nationale. Mener une recherche sur un segment aussi important présente sans conteste un intérêt et particulièrement lorsque cette recherche porte sur l'innovation, facteur important de survie de l'entreprise.

En effet, les PME du BTP sont caractérisées par leur manque de structurelle organisationnelle formelle (SOME, 2019). Mener des recherches sur l'innovation organisationnelle des PME du BTP ressemble à 'un épée dans l'eau'. Or les PME représentent plus de 90\% des entreprises au Burkina, singulièrement les entreprises du BTP à elles- mêmes ont une proportion de plus de 70\% (MESS, 2011). Malgré ces chiffres impressionnants, les BTP peinent à exécuter les travaux de qualité dans les délais, à respecter les cahiers de charges, à assurer un équilibre financier pour faire face aux charges. Cependant, les recherches sur les PME portent généralement sur les difficultés de financement, les difficultés d'accès aux marchés publics. Il nous semble original de jeter un regard sur la notion d'innovation et de performance dans cette fraction importante d'entreprises que sont les PME. D'où l'originalité et l'intérêt de ce sujet.

\section{Objectifs de la recherche}

Toute recherche doit avoir un objectif, ce qui permet au chercheur de mesurer le niveau d'atteinte de ses résultats. C'est ainsi que dans cette recherche, nous nous sommes fixé comme objectif de mesurer l'influence de certains facteurs internes sur le succès de l'innovation dans les PME du BTP.

Pour atteindre les objectifs, une revue de littérature des recherches antérieures sur la problématique est indispensable. 


\section{Revue de littérature}

L'Afrique se distingue par deux groupes d'entreprises : les grandes entreprises dominées en général par des multinationales et quelques entreprises publiques d'une part et les PME dont le capital appartient en totalité aux privés africains d'autre part. C'est ce dernier groupe d'entreprises qui nous intéresse dans cet article. Ces entreprises sont caractérisées par une organisation hybride fortement influencée par le pouvoir du dirigeant (Ngok \& Kombou, 2006).

Les recherches indiquent que ces entreprises manquent cruellement de personnel compétent capable de conduire des changements (SOME, 2019). L'innovation étant considérée comme la rupture d'avec les habitudes du passé, toute innovation, me semble -t-il ne peut réussir sans celle portant sur l'organisation et le mangement.

\subsection{Retour sur les typologies de l'innovation}

Plusieurs approches sont possibles en matière d'analyse de l'innovation dans l'entreprise. En mettant l'accent sur l'innovation apportée aux produits ou au marché, on parle d'innovation d'amélioration, incrémentale, opérationnelle et d'innovation de rupture (innovation disruptive). Ces types d'innovation sont la conséquence d'une innovation technologique et/ou innovation organisationnelle et managériales.

\subsubsection{L'innovation technologique}

De façon générale, l'innovation se définit comme l'ensemble des démarches scientifiques, technologiques, organisationnelles, financières et commerciales qui aboutissent, ou sont censées aboutir, à la réalisation de produits ou procédés technologiquement nouveaux ou améliorés (OCDE, 2007). Cette définition prend en compte les cinq types ${ }^{2}$ d'innovation $^{2}$ développés par Schumpeter en 1912.

L'innovation technologique relève du progrès scientifique et technique. Ce type d'innovation consiste à adopter une nouvelle technologie qui intervient directement dans l'amélioration des caractéristiques du produit final ou du service offert ou même des méthodes de travail. Elle se distingue des autres types d'innovation qui ne relèvent pas du progrès scientifique telle que l'innovation commerciale ou l'innovation organisationnelle.

\subsubsection{L'innovation organisationnelle et managériale}

Contrairement à l'innovation technologique qui semble trouver un consensus dans sa définition auprès des chercheurs, le concept de l'innovation organisationnelle, bien que largement

\footnotetext{
2 Joseph Schumpeter (1850-1934); considéré comme le père fondateur de l'innovation a distingué cinq types d'innovation : innovation de produits, innovation de procédés, innovation commerciale, les nouveaux types d'organisation, la découverte de nouvelle sources de matière $1^{\text {ère }}$ ou d'énergie
} 
développement dans la littérature ne rencontre pas l'assentiment de tous les chercheurs quant à sa définition.

L'innovation d'organisation repose sur un changement dans les pratiques de l'entreprise, dans l'organisation du lieu de travail ou dans la manière dont les relations extérieures sont gérées. Dans les anciens modèles de l'innovation, le changement organisationnel était vu comme une réaction face au progrès technique. Aujourd'hui au contraire, l'innovation organisationnelle tend à être considérée comme un préalable à l'innovation technologique (OCDE, 2005). La formation des salariés, la distribution des responsabilités et la structuration de la prise de décision, ou encore l'intégration de partenaires ou de clients dans les processus de l'entreprise sont des exemples d'innovation organisationnelle.

\subsubsection{Les approches définitionnelles de l'innovation organisationnelle}

Nous retenons ici deux définitions: celles issues des travaux Birkinshaw et al. (2008) et Damanpour et Aravind (2012).

Pour Birkinshaw et al. L'innovation organisationnelle (qu'ils nomment « innovation management») est définie comme « la génération et l'implémentation de pratiques, procédés, structures ou techniques de management qui sont nouveaux pour l'état de l'art et destinés à favoriser l'atteinte des objectifs organisationnels » (p. 829).

Quant à Damanpour et Aravind (2012), ils définissent l'innovation organisationnelle (qu'ils nomment» «innovation managériale ») comme «les nouvelles approches en matière de connaissances pour réaliser le travail de management et les nouveaux procèdes qui produisent des changements dans la stratégie, la structure, les procèdes administratifs et les systèmes» (p. 429).

Ces définitions font apparaitre deux points communs : le caractère multidimensionnel de l'innovation organisationnelle (qui englobe des pratiques, procédés, structures, techniques et approches) et le concept central de nouveauté (Dubouloz, 2014).

Les deux définitions ne font pas non plus allusion à la notion de l'innovation technologique.

Selon Williamson (1975), l'innovation organisationnelle inclut aussi bien les changements de formes organisationnelles que les systèmes et procèdes organisationnels, tels que la comptabilité analytique, l'organisation des plannings de travail, la négociation collective ou la gestion du personnel. De cette définition, nous retenons que l'innovation organisationnelle concerne plutôt les systèmes de gestion (gestion des connaissances par exemple), l'organisation du travail (méthode de travail, organisation du travail (méthode de travail, organigramme, travail collaboratif) et les relations extérieures de l'entreprise (relation avec les fournisseurs et autres partenaires). 
En ce qui concerne l'innovation managériale, c'est une forme d'innovation organisationnelle qui se focalise sur la manière dont se distribuent l'information, le pouvoir et le contrôle au sein de l'entreprise.

Au regard de ces deux approches, l'on se pose la question suivante: l'innovation organisationnelle et managériale est-elle possible dans le cas des entreprises du PME du secteur BTP au Burkina Faso?

La question ainsi posée semble être intéressant dans la mesure où les PME évoluent dans une sorte d'hybridation de leur formation organisationnelle et un système de décision centré sur la personne chef d'entreprises. La communication (ascendante et descendante) est pratiquement inexistante. Or, les études montrent que les PME innovent (SOME, 2019). Il s'agit alors plutôt du niveau de succès des innovations dans les PME vue le manque d'implication organisationnelle sus décrit.

\subsection{L'analyse de l'innovation organisationnelle et managériale par la théorie des capacités dynamiques ${ }^{3}$}

L'approche basée sur les capacités dynamiques a été développée à la suite de l'approche ressources- base-view (RBV) pour apporter une perspective plus dynamique à la stratégie (Gulsun, 2012).

Il faut noter cependant que l'approche capacités dynamiques complète la RBV en étudiant les manières dont les entreprises peuvent maintenir leurs avantages concurrentiels. Elle est basée sur le renouvellement permanent des compétences.

L'approche par les capacités dynamiques ou la Dynamic Capability View (DCV) s'impose de plus en plus comme le cadre théorique de référence en ce qui concerne l'analyse de l'avantage concurrentiel durable des firmes Geoffroy Labrouche (2014).

Elles se caractérisent donc par la capacité d'une firme à reconfigurer sa base de ressources pour faire face aux changements de l'environnement.

Les capacités dynamiques sont imbriquées dans les processus, les méthodes appliquées, les routines organisationnelles ou stratégiques :

elles sont le fruit de l'apprentissage organisationnel par expérimentation et répétition, par exemple : capacité à nouer des alliances avec le monde de la recherche, puis capacité à gérer

3 Le terme capacité dynamique est apparu pour la lère fois dans l'article de Teece et Pisano en 1994 qui la définit comme « le sous-ensemble des compétences / capacités qui permet à la firme de créer de nouveaux produits et process et de répondre aux circonstances changeantes du marché ». 
- des projets de recherche, puis capacité à valoriser ces projets. Elles rendent ainsi possible la reconfiguration des ressources de l'entreprise (capacité à acquérir, capacité à abandonner, capacité à maitriser la chaine de valeur) ;

- Elles permettent de faire face à des changements rapides de l'environnement, capacités de s'adapter aux nouvelles réalités des marchés. Elles construisent une configuration particulière de ressources, difficile à imiter, qui peut fournir un avantage concurrentiel Teece (2010).

Il faut noter cependant que l'apparition de la notion de capacité dynamique à fait naitre beaucoup de polémiques dans la recherche quelquefois considérées comme des limites. Ce qui a fait naitre deux tendances, certains considérés comme des opposants et d'autres comme des défenseurs du concept (Gulsun, 2012) ${ }^{4}$.

Pour les uns il s'agit d'un concept flou, difficile à opérationnaliser et sans fondement théorique. Ces derniers préconisent alors son abandon pur et simple et de se focaliser sur des concepts existant tel que la stratégie d'adaptation de la firme ou le changement stratégique de la firme (Gulsun, 2012).

Pour les autres, il s'agit d'un concept en plein développement, d'où l'utilité du débat sur son fondement théorique. La capacité dynamique a un grand apport au management stratégique dès que les recherches de clarification et d'opérationnalisation du concept se développent. C'est ainsi qu'en 1997, Teece et Pisano accompagné de Shuen (cités dans Gulsun, 2012) ont adopté la définition des capacités dynamiques comme « l'aptitude d'une firme à intégrer, construire et reconfigurer des compétences internes et externes en réponses aux environnements en évolution constante ». Selon ces auteurs, le terme « capacité » met l'accent sur le rôle du management stratégique à adapter, intégrer et reconfigurer, de manière appropriée, les compétences et ressources organisationnelles ainsi que les compétences fonctionnelles en réponse aux changements de l'environnement. Le terme «dynamique » fait, quant à lui, référence à la capacité à renouveler les compétences de manière à être en phase avec l'environnement, dans la mesure où des réponses créatives sont nécessaires dans certaines circonstances notamment lorsque le temps de mise sur le marché est critique, le taux de changement technologique est

\footnotetext{
${ }^{4}$ Gulsun Altintas dans un article publié en 2012 sous le titre «Les Capacités Dynamiques : une nouvelle perspective de recherche pour le Management Stratégique ? a présenté une analyse profonde des oppositions et défenseurs de cette approche
} 
élevé et la nature de la concurrence et des marchés est difficile à déterminer (Gulsun, 2012). Il s'agit là de la capacité à innover de la firme.

L'innovation est considérée de nos jours comme un élément de survie pour les entreprises, tout comme les capacités dynamiques dont l'objet pour l'entreprise est de préserver une place concurrentielle sur le marché. Il apparait alors un lien entre les capacités dynamiques et l'innovation.

\subsection{Les propositions de recherche}

Notre question de recherche est ainsi formulée: Quels sont les obstacles au succès de l'innovation organisationnelle et managériale dans les PME du BTP au Burkina Faso ?

Pour répondre à cette question centrale, nous avons émis deux (2) propositions de recherche.

P1 : L'instauration d'une bonne communication interne est un facteur de succès de l'innovation organisationnelle et managériale

La bonne communication permet une saine circulation de l'information dans l'entreprise. Elle permet de partager la vision de l'entreprise et de mettre tous les acteurs à un même niveau d'information. Plus l'information est partagée, plus chacun se sentira interpelés dans les taches qui sont les siennes.

Nous avons associé à cette proposition de recherche deux variables : les outils oraux de communication ayant comme indicateur l'utilisation du téléphone et la tenue de réunions de service.

La deuxième variable est les outils écrits de communication. Les indicateurs retenus à ce niveau sont : les notes de services et l'utilisation de l'internet.

\section{P2: La qualité des ressources humaines influence positivement sur l'innovation organisationnelle et managériale}

Les ressources humaines sont un facteur indispensable dans le fonctionnement de toute organisation, il l'est davantage lorsque l'entreprise dispose de ressources humaines de qualité. Les ressources humaines de qualité passent d'abord par un processus de recrutement transparent privilégiant les compétences, mais aussi par la formation continue. La formation continue permet de mettre à jour les compétences du personnel par rapport aux nouvelles technologies. Les variables retenues pour cette proposition de recherche sont : la motivation et la compétence du personnel.

La motivation a pour indicateurs associés le pourcentage de chef d'entreprises qui ont déclaré être satisfaits de la motivation de leur personnel. La compétence est également mesurée les déclarations des chefs d'entreprises. 
L'analyse des verbatims des MOD et des MOE viendra compléter les résultats des chefs d'entreprises.

Ces propositions de recherche nous ont permis construire un modèle de recherche à partir duquel nous allons mener nos analyses.

\subsubsection{Construction du modèle de recherche}

Les propositions de recherche décrites plus haut nous conduit à la construction du modèle de recherche suivant :

\section{L'instauration d'une bonne communication interne est un facteur de succès de l'innovation organisationnelle et managériale}

La qualité des ressources humaines influence positivement sur l'innovation organisationnelle et managériale
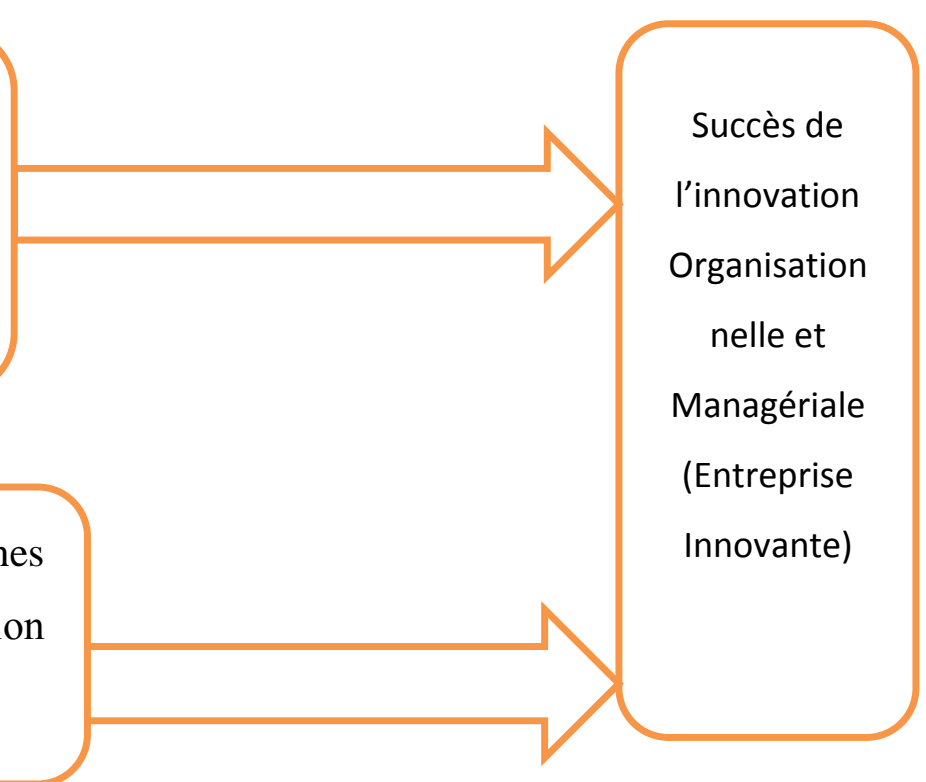

\section{Source : Notre construction}

Le modèle indique l'influence de nos deux propositions de recherche sur le succès de l'IOM de l'entreprise.

\section{Orientation méthodologie de la recherche}

Dans toute recherche, la précision du positionnement épistémologique et du type de recherche est indispensable pour le chercheur.

\subsection{Positionnement épistémologique et type de recherche}

Cette recherche s'inscrit dans une démarche de type qualitatif, ce qui nous conduit à opter soit pour le constructivisme soit pour l'interpretativisme. Dans ces deux paradigmes, on se pose la question du comment et du pourquoi. En recherchant les motivations (le pourquoi) comme le fait l'approche interprétativiste, on introduit la complexité psychologique dans la réflexion menée par rapport à l'objet de recherche. Cela implique de rester très proche des discours des acteurs que l'on cherche à analyser.

De son côté, l'approche constructiviste recherche les finalités des actions menées par les acteurs dans l'organisation. Elle introduit donc la complexité systémique dans l'ensemble des 
interdépendances imbriquées. Il y a de ce fait incompatibilité avec les techniques économétriques qui ne s'appliquent que sur des phénomènes dont l'analyse permet d'isoler rigoureusement et de manière transparente les interdépendances. Pour ces deux approches, les techniques quantitatives de recherche sont donc peu adaptées et la compréhension des phénomènes se traduit le plus souvent par l'analyse du discours des acteurs, champ d'investigation favorable aux méthodes qualitatives.

Notre démarche vise ici à comprendre comment les propositions de recherche citées plus haut peuvent - elles contribuer au succès de l'innovation organisationnelle et managériale, d'où le choix de notre positionnement constructiviste.

\subsection{Choix de l'échantillon et collecte de données}

Notre recherche se base une revue de littérature. Nous avons utilisé des données empiriques issues d'une enquête menées auprès de trente (30) entreprises du secteur du BTP et cinq (5) bureaux de contrôle ou maitrise d'œuvre (MOE) et de cinq (5) maitrise d'ouvrage délégués (MOD). Le choix de ces acteurs se justifie par le fait qu'il s'agit d'acteur incontournable dans le succès de l'acte de bâtir.

Figure 1 : Les interactions entre les principaux acteurs de l'acte de bâtir

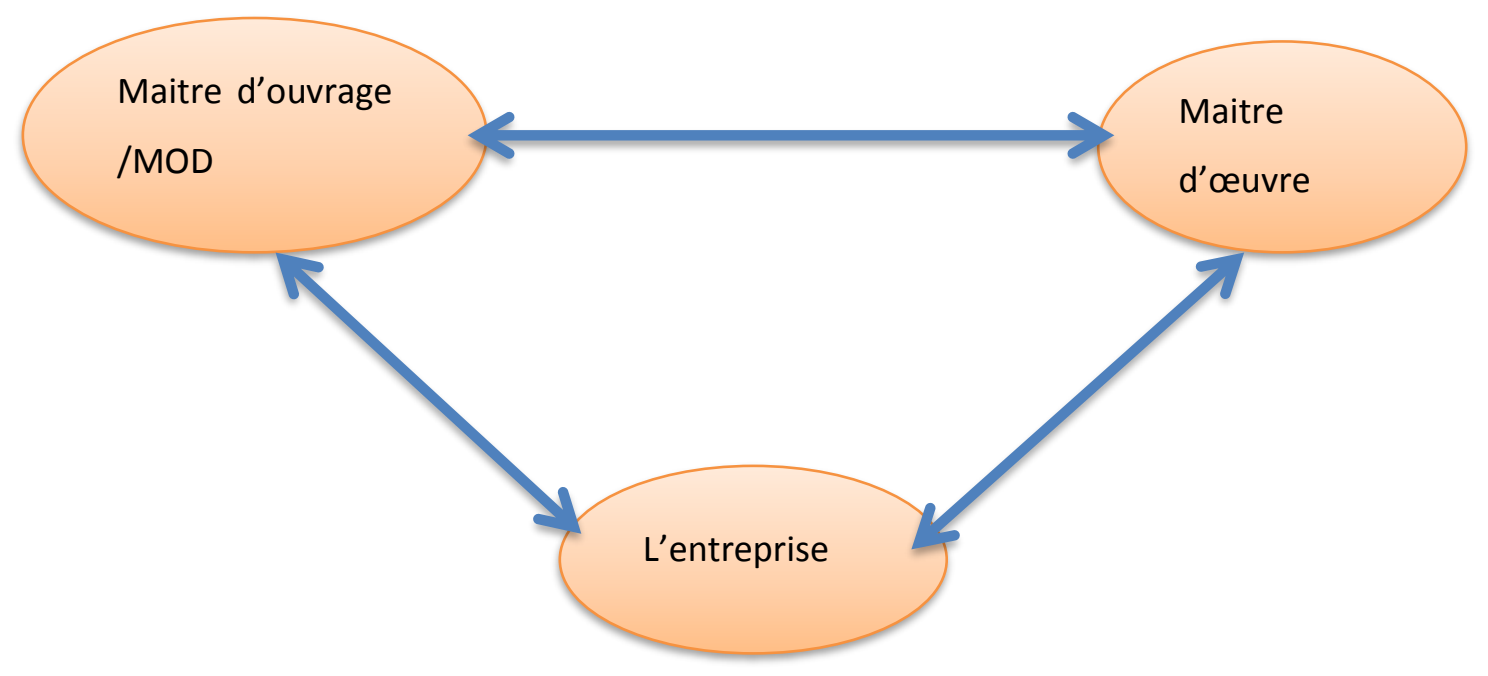

Source : Adapté de Prost, 2005 p14

La figure ci-dessus indique les principaux acteurs directs intervenant dans l'acte de construire. Elle indique les interactions réciproques entre les trois acteurs, ce qui justifie les flèches dans les deux sens.

Ces données ont été collectées entre juillet et octobre 2018. Elles ont été analysées dans le cadre d'une thèse en sciences en de gestion. 


\section{Résultats et discussion}

Les données collectées ont été regroupées en fonction de nos propositions de recherche que nous présentons comme suit.

\subsection{Présentation des résultats et analyses}

Nous présentons les résultats en fonction de nos propositions de recherche à partir des données des entreprises et de celles des bureaux d'études.

P1 : L'instauration d'une bonne communication interne est un facteur de succès de l'innovation organisationnelle et managériale

\section{* Les résultats obtenus auprès des entreprises du BTP}

Nous rappelons que dans la recherche de départ, un questionnaire a été soumis aux entreprises du BTP. Ce sont ces résultats que nous présentons ici.

Les outils de communication utilisés dans l'entreprise ont été divisés en deux groupes : les outils oraux de communication et les outils écrits de communication. Pour dérouler de bonnes innovations organisationnelles acceptées par tous, il faut que le personnel soit au même niveau d'information.

- La variable outils oraux de communication. Il a été mesuré le pourcentage d'entreprises qui tiennent des réunions de service, il est ressorti que seulement $47 \%$ des entreprises tiennent des réunions de service ( $27 \%$ très fréquemment et $20 \%$ fréquemment).

- En ce qui concerne les outils écrits de communication, l'étude a cherché à savoir si les entreprises communiquent à travers les notes de services. Les résultats montrent que seulement $24 \%$ des entreprises utilisent cet outil de communication, ce qui montre la prédominance de l'oralité dans nos entreprises. A contrario, l'étude indique que $87 \%$ des entreprises utilisent l'internet comme moyen de communication. Ce pourcentage est très proche de celui de l'utilisation du téléphone (94\%), il est à prendre avec beaucoup de prudence car une investigation approfondie par endroit à laisser comprendre que certaines personnes ont confondu l'utilisation du SMS à celui de l'internet.

\section{Les perceptions du système de communication par les MOD et MOE au sein des entreprises du BTP}

Un guide d'entretien a servi à recueillir la perception des Maitres d'ouvrage Délégué et des Maitres d'œuvre sur le système de communication dans les entreprises du BTP.

Les résultats montrent qu'il n'existe pas un système de communication permettant de partager l'information de façon équitable. 
Un enquêté déclarait ceci à la question de savoir si les entreprises ont des cadres de rencontres : « on ne parle pas de transparence dans les entreprises du BTP », « Rare sont les entreprises qui tiennent des réunions périodiques dans leur service, cela se sent à travers les contradictions entre personnel d'une même entreprise quand on fait les rencontres de chantiers », renchérit un autre.

Leur niveau de perception de la transparence dans les entreprises du BTP est apprécié à travers les verbatims suivants : «Beaucoup de choses sont tenues au secret», « l'entreprise est centrée sur le premier responsable qui ne communique pas assez avec les autres collaborateurs».

Ces résultats corroborent avec ceux obtenus auprès des chefs d'entreprises du BTP.

P2 : La qualité des ressources humaines influence positivement sur l'innovation organisationnelle et managériale

La qualité des ressources humaines nous semble indispensable pour la mise en œuvre d'une innovation organisationnelle et managériale. Les ressources humaines de qualité s'acquièrent selon deux volets : par le recrutement et par la formation continue.

\section{Résultats relatifs à la compétente des ressources humaines vue par les chefs d'entreprise du BTP}

Les entreprises affirment à 97\% qu'elles ont du personnel qualifié. Cette affirmation correspond au respect des cahiers des charges pour l'obtention des agréments techniques et à la satisfaction des conditions pour l'obtention des marchés. En effet, l'appartenance de l'entreprise à une catégorie donnée l'oblige à présenter un personnel qualifié répondant à ladite catégorie. Les entreprises qui ont des agréments valides sont celles qui répondent avoir du personnel qualifié. Aussi, le personnel technique présenté pour les soumissions aux appels d'offre n'est pas celui qui est sur le chantier.

La délégation de tache à un collaborateur est un facteur de motivation et de responsabilisation. Le collaborateur se sent responsable et a tendance à donner le meilleur de lui - même. Dans les PME du secteur du BTP au Burkina Faso, seulement 23\% des responsables délèguent les taches aux collaborateurs (SOME, 2019) au motif que le personnel n'est pas compétent.

Ce manque de délégation a été confirmé par les maitres d'œuvre et les maitres d'ouvrage à travers ces propos : «c'est le chef d'entreprise qui concentre tout, quand il n'est pas là tout est bloqué ».

\section{L'appréciation de la qualité et de la motivation des ressources humaines dans les BTP par les MOD et MOE}

Les données recueillies en relation avec la qualité des ressources humaines dans le BTP sont résumées à travers quelques verbatim. 
Il ressort de l'étude que les entreprises manquent du personnel permanent en leur sein :

«Beaucoup d'entreprises ont à peine une secrétaire, il y en a qui n'ont même pas de local».

« Des entreprises ont l'agrément de la catégorie B4, mais si vous arrivez, c'est la secrétaire et le patron, quand tu demandes et les autres, il vous répond qu'ils sont sur le terrain»5.

En ce qui concerne l'appréciation du niveau de motivation du personnel, pour les MOD et les MOE, le personnel n'est pas motivé :

«aucun plan de carrière dans les entreprises de BTP», «Les agents ne bénéficient pas de formation ».

Il existerait une certaine iniquité dans le traitement du personnel, ceci est justifié par les verbatims suivants : « le personnel n'est pas traité de la même façon »

«des ingénieurs sont moins payés par rapport à des techniciens sur des bases subjectives», « Mobilité très accrue des cadres».

« le personnel n'est pas valorisé, c'est le patron qui détient toutes les informations, ce qui explique les mouvements dans les entreprises».

« le personnel n'est pas traité de la même façon, souvent des techniciens sont mieux traités que des ingénieurs».

Ces verbatim illustrent fort bien la situation du personnel dans les PME du BTP.

\subsection{Analyse et interprétations des résultats}

Notre recherche vise à analyser quelques facteurs qui entravent la réussite de l'innovation dans les entreprises du BTP au Burkina Faso. Les résultats que nous avons obtenus ont été présentés suivant nos deux propositions de recherche en fonction des variables de mesure retenues. Ils seront également analysés et interpréter suivant le même procédé.

* P1 : L'instauration d'une bonne communication interne est un facteur de succès de l'innovation organisationnelle et managériale

L'analyse et l'interprétation des résultats liés à la communication se fait selon qu'il s'agit des outils oraux de communication (téléphones, réunions) ou les outils écrits (notes de service, internet).

Les résultats indiquent une forte dominance d'utilisation des moyens de l'oralité dans les entreprises du BTP. Ainsi, 94\% des entreprises utilisent le téléphone comme moyen de communication dans l'entreprise. Quant aux réunions de service, seulement $47 \%$ de tiennent des réunions. Le téléphone est un excellent moyen de communication qui permet d'atteindre le

\footnotetext{
${ }^{5}$ L'exercice de la profession d'entrepreneur du Bâtiment est soumis à une obtention préalable d'un agrément délivré par l'Etat autorisant à exécuter des travaux donnés. Ces agréments vont de B1 à B4.L'agrément B4 est le plus élevé dans le domaine du Bâtiment. Son titulaire peut exécuter toute sorte de travaux de bâtiment.
} 
plus rapidement possible sa cible, son inconvénient dans l'entreprise est qu'il ne permet pas de partager la même information de la même manière, l'information peut être sujette à interprétation et il peut être difficilement utilisé comme élément preuve du contenu de l'information transmise.

Les réunions de service ont l'avantage de partager les informations avec le personnel au même moment et de la même manière. C'est un cadre de rencontre qui permet aux entreprises de discuter du niveau d'avancement des travaux et de trouver des solutions aux goulots d'étranglement. L'absence de réunion de service ou de chantier est une grave faiblesse car c'est le lieu par excellence de communication des objectifs de l'entreprise et de l'évaluation du niveau d'avancement de la mise en œuvre des innovations. Ces résultats corroborent avec ceux de Ouédraogo (2005). Dans le cadre de son étude sur le projet de construction d'un immeuble $\mathrm{R}+5$, il est ressorti que les réunions de chantiers ne sont pas tenues.

En ce qui concerne les outils écrits de communication, deux ont essentiellement retenu notre attention dans cette recherche : il s'agit des notes de services et de l'internet. A ce niveau, les résultats indiquent que seulement $24 \%$ utilisent la note de service comme moyen de communication contre $87 \%$ pour ce qui est de l'internet. On observe que le taux d'utilisation de l'internet est assez proche de celui du téléphone, respectivement $87 \%$ et $96 \%$. Ce taux est cependant à prendre avec beaucoup de prudence. Le questionnement approfondi chez certains entrepreneurs a laissé transparaitre une confusion entre le SMS et l'internet.

Les résultats indiquent une faiblesse voir une absence de transparence dans la communication au sein des entreprises du BTP. Cela est confirmé par les entreprises elles-mêmes à travers l'étude quantitative et par les MOD e les MOE à travers l'étude qualitative.

Ces résultats sont en cohérence avec les pratiques dans les PME du Burkina Faso d'une manière générale et en particulier dans le BTP.

La PME est très centrée sur la personne du dirigeant. Il est le chef d'orchestre ; il assure la planification stratégique et opérationnelle, exécute, évalue et réoriente seul les actions de l'entreprise. L'absence de cadre de rencontre confirme que les projets d'innovation ne sont pas connus du personnel et par conséquent, il peut donc pas s'appliquer, ce qui constitue un obstacle au succès des innovations entreprises.

\section{* P2 : La qualité des ressources humaines influence positivement sur l'innovation organisationnelle et managériale}

Nous avons retenu la qualité des ressources humaines comme facteurs pouvant influencer sur le succès de l'innovation. 
Les résultats de la recherche indiquent que les PME du BTP manquent cruellement du personnel en nombre et qualité suffisant « le personnel de certaines entreprises se limitent à la secrétaire » disait un enquêté.

«L'entreprise, c'est avant tout des hommes, car on déjà vu des entreprises se monter sans argent, mais on en a jamais vu sans personnel » (Michael Halimi cité dans SOME, 2019. Ces propos illustrent bien la nécessité d'impliquer le personnel dans la gestion de l'entreprise et donc dans le succès de l'innovation.

Les résultats indiquent que $97 \%$ des entreprises affirment disposer du personnel de qualité. Cette affirmation est contrariée par les entreprises elles-mêmes lorsqu'elles affirment ne pas déléguer au motif qu'il n'existe pas de personnel qualifié.

Le manque de personnel qualifié est également confirmé par les MOE et les MOD. En effet, tout en reconnaissant l'absence de personnel qualifié sur le marché, ils insistent également que les entreprises parfois préfèrent le personnel sous-qualifiées au prétexte que le personnel qualifié coûterait cher. Le manque de qualification est observé sur le terrain dans l'exécution des marchés (ouvrage de mauvaise qualité en partie due au manque de qualification, retard dans l'exécution du à l'absence du personnel qualifié dans l'entreprise etc.).

Une fois le personnel entre dans l'entreprise, celle-ci doit lui assurer une formation continue pour une mise à jour de ces connaissances. Or à ce niveau seulement $33 \%$ affirment assurer une formation continue au personnel.

Le même constat est fait au Maroc à travers l'étude de Dikra El Maguiri en $2016^{6}$ «les différents constats et études soulevés et menées ces dernières années sur le sujet de la formation professionnelle continue (FPC) ou encore la formation en cours d'emploi FCE1, au Maroc affirment qu'il s'agit d'une fonction trop souvent négligée par les entreprises marocaines. Certaines y voient une charge sociale supplémentaire.... ».

Aussi, le personnel doit être motivé afin de donner le meilleur de lui - même. Nous avons donc retenu la variable formation continue et la variable motivation.

Les résultats indiquent que le personnel du BTP n'est pas motivé. En effet, 52\% des chefs d'entreprises affirment être satisfaits de la motivation de leur personnel. Cependant, pour les MOE et les MOD, le personnel manque de motivation dans les entreprises du BTP pour les raisons suivantes : absence de carrière, manque d'équité dans le traitement salarial. Ces résultats reflètent l'organisation générale de l'entreprise. En effet, les entreprises ne disposent pas de grille ou de politique de fixation de salaires. Des ingénieurs se retrouvent à un niveau de salaire

\footnotetext{
${ }^{6}$ Etude sur le développement et l'efficacité de « la formation en cours d'emploi » au sein de l'entreprise marocaine : Cas du secteur BTP au Maroc
} 
inférieur à celui d'un technicien par le simple fait d'affinité existant entre le chef d'entreprises et certains employés. Aussi, les cadres ne se sentent pas valorisés (absence de délégation de taches, absence de partage d'information).

Avec des ressources humaines insuffisantes, incompétentes ou démotivées, toute innovation est vouée à l'échec.

La convergence des résultats obtenus auprès des entrepreneurs et ceux issus des MOE et des MOD permettent de valider nos deux propositions de recherche.

Nous relevons cependant des limites dans cette recherche. En effet, cette recherche ne mesure pas le degré d'influence des différentes variables étudiées dans le succès ou dans l'échec des actions d'innovation de la PME.

\section{Conclusion}

Notre recherche a porté sur les facteurs de succès de l'innovation. C'est une recherche de type qualitatif basée sur les résultats de recherche auprès de 30 PME du BTP et de 10 bureaux d'études et de maitrise d'ouvrage déléguées d'une thèse. Il est ressorti que les entreprises qui ont innové ces trois dernières années ont vu leur chiffre d'affaires augmentés. Nous avons retenu comme facteurs influençant l'innovation la qualité de la circulation de l'information dans l'entreprise, la qualité des ressources humaines.

Nous retenons que dans le BTP, l'information n'est pas partagée de façon fiable. Cette conclusion est également tirée par Chafik Khalid en 2015 ${ }^{7}$. Pour cet auteur, le système d'information est peu formalisé dans les PME Marocaines. Aussi, les PME Marocaines sont sujettes à des facteurs de contingence comportementale liés du profil du dirigeants : le type de formation et le niveau d'étude (Chafik, 2015). Ces facteurs se retrouvent également dans les entreprises du BTP au Burkina Faso.

En effet, l'information est détenue par le chef d'entreprise qui la transmet de façon informelle à chaque agent, aucun cadre de rencontre n'existe dans l'entreprise. Ce qui fait dire à certains acteurs du BTP qu'il n'existe pas de transparence informationnelle dans les entreprises du BTP. Alors que l'innovation doit être partagée dans l'entreprise afin que chaque acteur se sente responsable. L'implication du personnel est indispensable pour le succès de l'innovation. La condition pour que le personnel s'implique, est qu'il doit être compétent et motivé. La compétence commence certes au niveau de l'acquisition des ressources humaines, mais elle se maintient à travers les formations continues. A ce niveau les entreprises affirment ne pas former

\footnotetext{
${ }^{7}$ La formalisation du système d'information des PME Marocaine : essai d'analyse des facteurs de contingence, mai 2015.
} 
leur personnel à $77 \%$. En termes de motivation seulement $52 \%$ des chefs d'entreprises affirment être satisfaits de la motivation de leur personnel au travail. Les autres acteurs de l'acte de construire (MOD et MOE) affirment que les PME du BTP ne disposent pas du personnel qualifié au regard de la qualité de travaux, et même quand il existe, il est démotivé à cause des facteurs tels que l'absence de carrière, le manque d'équité etc.

$\mathrm{Au}$ regard de ces résultats, nous avons conclu que les entreprises du BTP échouent dans l'innovation. Cependant, les facteurs que nous avons étudiés ne sont pas les seuls à même d'influencer sur l'innovation. Il serait alors important d'explorer d'autres facteurs qui viendront en complément de ceux ayant fait l'objet de la présente recherche

\section{BIBLIOGRAPHIE}

[1] Ayerbe-Machat, C. (2003, juin 3,4,5, et 6). Innovations technologique et organisationnelle au sein de PME innovants : complémentarité des processus, analyse comparative des mécanismes de diffusion. XIIème conférence de l'Association Internationale de Management Stratégique, les côtes de Carthage.

[2] Bakengela Shamba, P. (2007, Septembre). Existe-t-il un modèle spécifique du Management en Afrique ? le «management Africain» à l'épreuve des évidences empiriques. 18ème congrès de l'Association de Gestion des Ressources Humaines, p. Suisse.

[3] Birkinshaw, J., G. Hamel, et M. Mol (2008). Management Innovation, Academy of Management Review, $33: 4,825-845$.

[4] Bonnin D, \& Bedr, B. (2001). Développement du conseil en ergonomie : vers une prestation de conseil élargie destinée aux Petites, Petites et Moyennes Entreprises (TPE-PME). 36ème congrès de la Société d'Ergonomie de Langue Française.

[5] Brion, S. (2012). L'organisation comme levier de l'innovation. Gestion et Management, université de Grenoble alpes.

[6] Chafik, K (2015), la formalisation du système d'information des PME Marocaine : essai d'analyse des facteurs de contingence, mai 2015

[7] Damanpour F., Aravind D. (2012). « Managerial innovation : conceptions, processes, and antecedents », Management and organization Review, vol.8, $\mathrm{n}^{\circ} 2, \mathrm{p} .423-454$

[8] Deltour, F. (2014, mai 26-28). Innovation et performance des PME : une approche par la contribution des technologies de l'information. AIMS, XXIII Conférence Internationale de Management Stratégique.

[9] Dikra El,M. (2016). Étude sur le développement et l'efficacité de la formation en cours d'emploi au sein de l'entreprise marocaine : cas du secteur BTP au Maroc, Université Hassan II-Casablanca, Maroc. International Journal of innovation and Applied Studies, Vol.15 n² Apr., pp307-318.

[10] Dubouloz, S. (2013). Les barrières à l'innovation organisationnelle : le cas du Lean Management. Management. Management international.

[11] Dubouloz, S. (2013). L'innovation organisationnelle : antécédents et complémentarité, une approche intégrative appliquée au Lean Management, thèse de doctorant en Sciences de Gestion, Université de Grenoble ; pp440.

[12] Gulsun, A (2012). Les Capacités Dynamiques : une nouvelle perspective de recherche pour le Management Stratégique ? Institut National de la statistique et de la démographie. (2010). Recensement Statistique des Entreprises, Burkina Faso. 
[13] Maison de l'Entreprise du Burkina (2011). Etude sur le profil des créateurs d'entreprises et d'évaluation de mortalité des nouvelles entreprises. Ouagadougou.

[14] Maison de l'Entreprise du Burkina (2015). Étude sur la mortalité des entreprises créées dans les CEFORES du Burkina entre 2006 et 2013. Ouagadougou.

[15] Sylla, K (2013). Les déterminants de la stagnation des micros et petites entreprises béninoises. Revue africaine de gestion, $\mathrm{N}^{\circ} 4$ avril.

[16] Maltese, L. (2007, Juin 6-9). Spécificités des actifs et aménagement des évènements sportifs : proposition d'une nouvelle approche méthodologique et analytique. XVème conférence Internationale de Management Stratégique.

[17] MESS (2011). Formation dans le secteur du Bâtiment et des Travaux publics au Burkina Faso Prospective et propositions

[18] Moungou Mbenda, Sabine Patricia (2007). Le pouvoir du dirigeant dans la relation d'agence : Cas des entreprises publiques au Cameroun, XXVIème Conférence Internationale de Management Stratégique ; Lyon 7-9 juin.

[19] Ngok Evina, J.F. et L. Kombou (2006). L'influence du pouvoir du dirigeant sur la structure de l'entreprise : une étude à partir des entreprises camerounaises, La Revue des Sciences de Gestion,219,89-98

[20] Labrouche, G. (2014, Mai 26-28). Les capacités dynamiques : un concept multidimensionnel en construction. XXIIIème, conférence Internationale de Management Stratégique.

[21] Le Roy F., Robert M., Giuliani P. (2012). « Quels liens entre l'innovation technologique et managériale ? Pour une distinction entre l'innovation produit et l'innovation procédé », XXIème conférence de l'Association Internationale de Management Stratégique, Lille,4-6 juin

[22] OCDE, (2007).la mesure des activités scientifiques et technologiques Principes directeurs proposés pour le recueil et l'interprétation des Données sur l'innovation technologique, manuel d'Oslo, pp103

[23] Ouédraogo, F. (2010). Analyse de la gestion de chantier : cas des travaux de construction et de butimage de l'interconnexion des routes nationales RN1-RN4, lot 1 ; tronçon, RN1. Mémoire pour l'obtention du Master en ingénierie de l'eau et de l'environnement, option génie civil.21E, Ouagadougou.

[24] Sawadogo, R. (2013). Organisation d'un chantier de construction du complexe administratif $R+5$ avec soussol à usage de bureau dans la ZACA de Ouagadougou, Master d'ingénierie de l'eau et de l'Environnement, option génie civil. Institut international d'Ingénierie et de l'Eau.

[25] SOME, L. (2016). Contribution à la détermination et à l'analyse des obstacles à la performance des PME au Burkina Faso : cas des PME du secteur du BTP, Université Aube Nouvelle, Mémoire de master recherche.

[26] SOME, L. (2019). Exploration des facteurs de contre-performance des PME du secteur du BTP au Burkina Faso, Université Aube nouvelle, thèse de doctorat en Sciences de gestion. Ouagadougou.

[27] Teece, D.J. (2010). Business Models, Business Strategy and Innovation. Lon Range Planning, pp.172.194.

[28] D.J. (1980). « The diffusion of an administrative innovation », Management Science, vol.26, n 5 , p.464-470. 